\title{
Multiobjective Optimized Routing Protocol for VANETs
}

\author{
Taqwa O. Fahad $\mathbb{D D}^{1}$ and Abduladhem A. Ali ${ }^{2}$ \\ ${ }^{1}$ University of Technology, Iraq \\ ${ }^{2}$ Department of Computer Engineering, University of Basra, Iraq \\ Correspondence should be addressed to Taqwa O. Fahad; taqwa.odey@gmail.com
}

Received 25 May 2018; Revised 30 October 2018; Accepted 26 November 2018; Published 11 December 2018

Guest Editor: Jiefeng Liu

Copyright (c) 2018 Taqwa O. Fahad and Abduladhem A. Ali. This is an open access article distributed under the Creative Commons Attribution License, which permits unrestricted use, distribution, and reproduction in any medium, provided the original work is properly cited.

\begin{abstract}
Vehicular ad hoc network (VANET) routing protocols have been attracting a considerable attention of both research and industrial communities, due to their significant role in intelligent transportation system applications. The present paper adopts an optimized integrated multicast, multicriteria, adaptive route lifetime as a routing protocol for VANETs. Whereby only an optimal subset of neighbor vehicles is chosen to relay route request (RREQ) messages based on distance, direction, speed, and future direction information in a combined sender-receiver manner. Among those selected optimal paths for route discovery, the best route with lowest cost will be chosen for forwarding data packets for a specified duration assigned depending on the obtained cost and number of intermediate vehicles of that route. Fuzzy controllers were employed to assess routes' costs and their lifetimes. Furthermore, artificial bee colony $(\mathrm{ABC})$ algorithm was used to concurrently optimize all used fuzzy systems and obtain the optimal highest rank of links' cost values within which the neighbors could be selected as relay nodes in route discovery process. Simulation results prove that the proposed routing scheme significantly improves the network performance in both urban and highway scenarios, under different situations of vehicle density.
\end{abstract}

\section{Introduction}

The vehicular networks $[1,2]$ are a promising technology that enables smart vehicles to exchange wireless messages among themselves, in order to achieve more convenient and safer transportation system. These messages may include information about traffic flow condition, adaptive trip assistance, warnings and alarms, and parking or gas station availability, as well as infotainment applications.

These numerous VANET applications substantially rely on VANET routing protocols $[3,4]$, which are originated from preceding wireless mobile ad hoc network (MANET) algorithms but have been extensively reformed to cope with VANET characteristics and requirements.

The unique VANET characteristics of highly mobile vehicles, limited coverage area, intermittently wireless connection, and traffic density, in addition to restriction of movement within road patterns and traffic rules, make providing more reliable and efficient routing protocol for these challenging networks is still an open research topic; some of these recently related studies attend.
Li et al. [5] propose an adaptive QoS routing by using terminal intersections and an ant colony algorithm to search the optimal route based on connectivity probability, packet delivery ratio, and delay.

Al-Kharasani et al. [6] propose a particle swarm optimization tuned optimized link state routing protocol considering multiple objectives of throughput, packet delivery ratio, delay, and normalized routing overhead to evaluate the fitness function.

Latif et al. [7] offer an algorithm to select the next forwarder vehicle by using multicriteria based mathematical formulation considering direction, position, and distance of vehicles with respect to source vehicle.

Zhang et al. [8] suggest multicast routing protocol based on microartificial bee colony, in which energy consumption and transmission delay are considered to measure the quality.

Miao et al. [9] present fuzzy logic based routing scheme that forward broadcasted packets depending on fuzzifying two factors regarding distance and time delay.

Nabil et al. [10] propose a scheme to select the most stable route and predict its lifetime by calculating the link stability 
time of vehicle's neighbors according to mathematical equations taken into account four cases of same or opposite direction of movement with same velocity or not.

However, the essential uniqueness of the current study represents by addressing the main three critical issues of routing in VANET in associative form; the first problem is the blind broadcasting of route request (RREQ) messages in route discovery phase, which result in high control messages overhead and packet collision problem especially in high dense network, or even in a sparse network with high packet transmission rate.

While the second issue regards high route breakage as a result of the omission of some affecting criteria in route selection process, resulting in high number of error messages and lost data packets.

Whereas the third issue treats the dilemma of using fixed route lifetime, regardless of the situation of the selected route, which might lead to disrupt some valid routes or consider some others as valid while they were broken.

Even though there are a number of works concerning the above first two problems [7-9, 11-13], they mostly consider only two or three factors in their studies. And some are completely based on sender to specify the relay nodes [11, 12], which typically require exchanging periodic hello messages, while the others are completely based on receiver to determine whether to rebroadcast the packet or not $[9,13]$, and as a consequence some redundant broadcasts are still not eliminated.

On the other hand, few works attend the third mentioned dilemma of routing in VANET environment [10, 14]; they mainly depend on mathematical models that take into account only two factors.

Particularly, this proposed protocol incorporates the principles of fuzzy systems to improve the decision-making processes of ad hoc on demand distance vector (AODV) routing protocol for vehicular network environment by evaluating the link cost of every neighbor in a combined sender-receiver fashion, taking the information of distance and direction into account at sender, in addition to considering speed and future direction information at receiver. This is in order to select only a subset of optimal relay neighbors for route discovery process, as well as select the best route for carrying data. Additionally, it could predict the lifetime of each selected route considering its obtained cost, besides number of its involved hops. Moreover, ABC optimized algorithm is used to generate the proposed fuzzy memberships' vertices and rules, aside from thresholds of selected relay neighbors' link cost.

More specifically, the main contribution keys of the proposed scheme in the present work can be summarized as follows:

(i) Three-step optimization using fuzzy systems in route discovery phase, regarding choosing relay nodes and appropriate route considering the most effective stability factors, in addition to specifying each route lifetime.

(ii) Combined sender-receiver-based multicast routing protocol without the overhead of using periodic Hello packets, as well as mitigating the shortcoming of completely receiver-based forwarding decider.

(iii) A novel stability factor regarding next direction of vehicles considered in route discovery phase, without transferring this datum to keep security of vehicles' future movements.

(iv) Multiple factors of distance, direction, speed, future direction, and number of hops considered in the proposed scheme.

(v) Automatic-extracted fuzzy controllers using artificial bee colony optimization.

(vi) Four objectives of optimization process considered: maximizing packet delivery ratio and throughput, and minimizing delay and number of control packets.

The rest of the paper is organized as follows: Section 2 describes the optimization algorithm used in the present study. Section 3 gives details about the proposed routing scheme. Simulation parameters and comparative results are presented in Section 4. Finally, conclusions and suggested future works are conferred in Section 5.

\section{Employed Optimization Method}

2.1. Artificial Bee Colony (ABC) Optimization. ABC optimization [15-17] is a swarm intelligent based scheme that simulates the foraging behaviour of honey bee colonies.

It is simple and flexible algorithm, requiring few parameters to be tuned; moreover, it could be hybridized with other intelligent algorithms easily.

Furthermore, many studies [18-20] have proven the efficiency and accuracy of using $A B C$ optimization and hybridization over many other popular optimization methods.

The general structure of $\mathrm{ABC}$ optimization algorithm consists of three groups of artificial bees: employed, onlooker, and scout bees.

Each employed bee is associated with a food source (possible solution) and shares the position information and nectar amount (quality or fitness cost) of these sources with onlooker bees for further processing. The onlooker bees decide the best profitable source food according to a probability selection process. When a food source does not improve up to a predefined number of trials, the food source is rejected by the bees and the corresponding employed bee becomes a scout bee.

More details about $\mathrm{ABC}$ algorithm are given below:

First, let $x_{i}=\left[x_{i 1}, x_{i 2}, \ldots, x_{i D}\right]$ denote the $i^{\text {th }}$ solution in the population, where $i=1,2, \ldots, S N$; however, $S N$ reflects the size of solutions in the population and is taken as half of the bee colony (equals number of employed bees or onlooker bees), while $D$ represents the number of optimization parameters.

2.1.1. ABC Initialization Phase. The $\mathrm{ABC}$ generates a randomly distributed initial population of $S N$ solutions within 


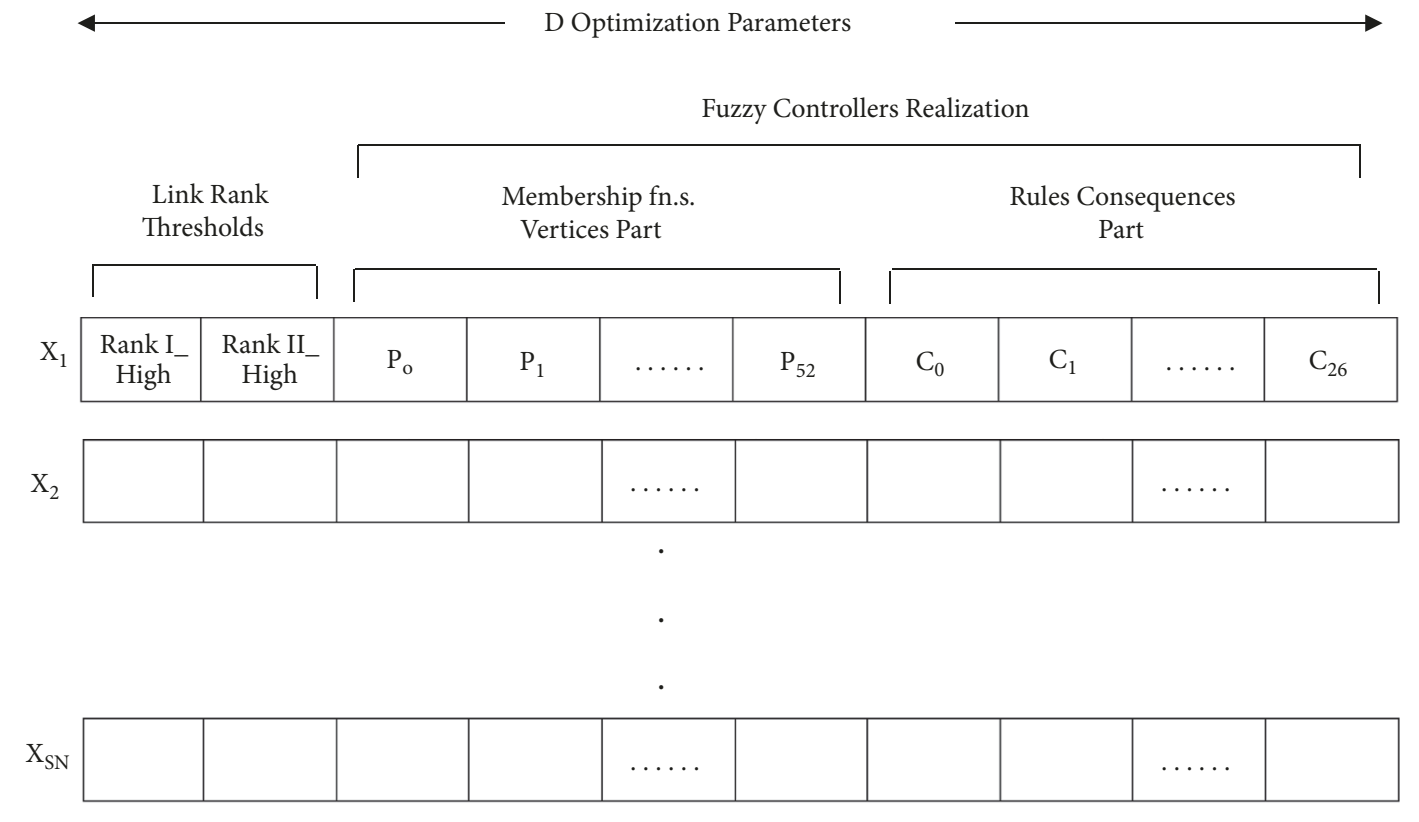

FiguRE 1: Food sources structure.

the range of boundaries according to the following equation:

$$
x_{i j}=x_{j}^{\min }+\left(x_{j}^{\max }-x_{j}^{\min }\right) * \operatorname{rand}(0,1)
$$
$x_{i j}$.

where $x_{j}^{\min }$ and $x_{j}^{\max }$ are the lower and upper bounds of

After solutions generation, the fitness function fit $_{i}$ for all initial solutions in the population is evaluated.

2.1.2. ABC Employed Bee Phase. In this phase, each solution is assigned to an employee bee, which produces a modification on the position of the solution as follows:

$$
v_{i j}=x_{i j}+\varphi_{i j} \cdot\left(x_{k j}-x_{i j}\right)
$$

where $v_{i j}$ is the mutant solution of the original solution, $\varphi_{i j}$ is a random number in the range $[-1,1]$, and $\mathrm{k}$ is a neighbor of $\mathrm{i}, \mathrm{k} \neq \mathrm{i}$.

After that, a greedy selection is performed by the employed bee between the original solution and its mutant according to their fitness evaluation values.

2.1.3. Probability Calculation Phase. For each solution, a probability value $p_{i}$ which is proportional to its quality is calculated as

$$
p_{i}=\frac{f i t_{i}}{\sum_{n=1}^{S N} f i t_{n}}
$$

2.1.4. ABC Onlooker Bee Phase. The onlooker bee evaluates the information taken from the employed bees and selects the solution with the highest probability value. Next, the same as employed phase mechanism of position adjustment and greedy selection are applied by the onlooker bees to memorize the better solutions.
2.1.5. ABC Scout Bee Phase. If a solution does not improve up to a predefined number of trials (exceeds a control parameter called Limit), the solution is abandoned, and its corresponding employed bee becomes a scout bee which replaces it with a new randomly solution using (1).

The execution of the above four phases will be repeated until reaching the maximum number of cycles for search, and the best obtained solution is given as the optimized output.

2.2. ABC Optimized Fuzzy System. Fuzzy logic [21, 22] has been widely employed for supporting many intelligent based real world systems especially under imprecise and uncertain information.

Typically, the knowledge base unit which consists of data base and rule base represents the heart of any fuzzy system.

However, the manual generation of the fuzzy knowledge base depending on system knowledge and trial and error process is a very tedious and time consuming task. Moreover, it does not guarantee the construction of an optimal system.

As a solution, ABC optimization is proposed to automatically extract the optimal rule set, with "do not care" condition exploration for more compact rules acquiring and tuning the membership functions for all the proposed fuzzy controllers.

Since all the proposed fuzzy controllers are depended, a collection of all membership function vertices for fuzzy inputs and outputs, in addition to linguistics for consequents of the rules, is encoded into the food source as shown in Figure 1 to guarantee the simultaneous upgrowth of the whole model parameters within the given search space.

In order to reduce number of optimization parameters to speed up the optimization process, the membership functions which were used for the proposed fuzzy controllers were set to triangular type, since it has proven more efficiency than other types in such network model [23-26]. 


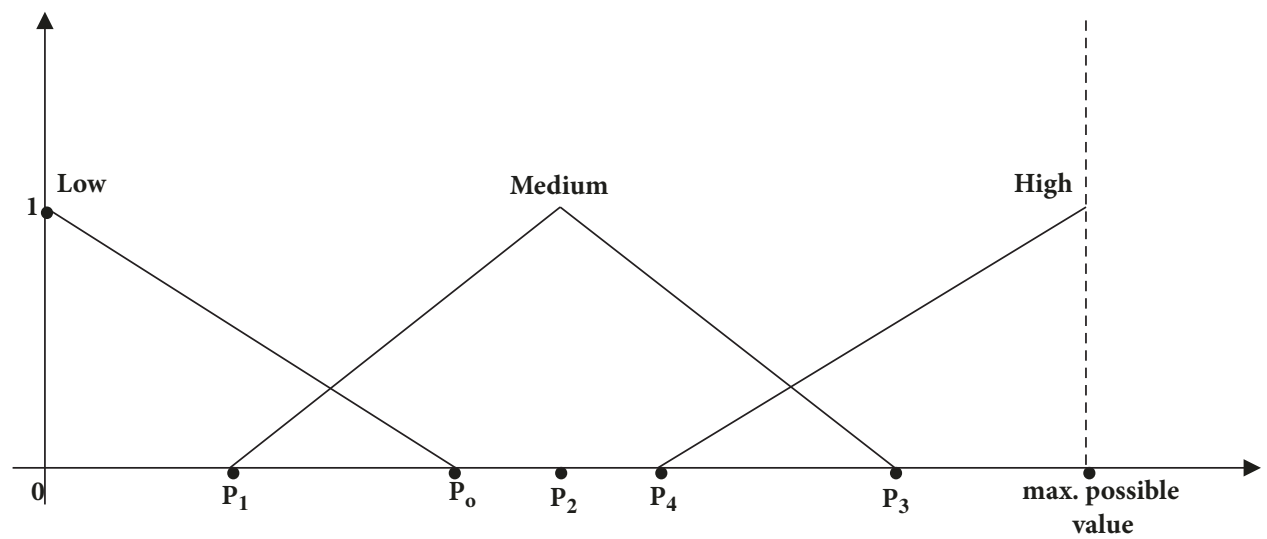

Figure 2: Fuzzy input optimized membership points.

Three linguistic terms were associated with each fuzzy input (Low, Medium, and High) and five for the output (Very Low, Low, Medium, High, and Very High) to get more accuracy.

As will be presented in next section, most fuzzy inputs were put in a ratio form, so that the first and last membership points were fixed and did not need to optimize. This gives a total of five membership optimized points which are required to represent each fuzzy input as shown in Figure 2.

Similar to the above mechanism, a total of eleven membership optimized points are required to represent the first two fuzzy controllers output, and an extra point are added in the last fuzzy controller output to get the optimal maximum route lifetime point.

In other respect, to generate the optimal rule base set, the fuzzy output linguistic terms are coded into real numbers from 1 to 5 , and ' 0 ' value is used to represent the rule absence. Hence, each rule antecedent may take an integer value ranging from 0 to 5 , such that ' 1 ' represents 'Very Low', '2' represents 'Low', and so on.

Furthermore, the objective of the optimization process in this work is to explore the best fuzzy models besides link rank thresholds that would be incorporated in the proposed routing algorithm to meet the best network performance outcomes through multiple objectives of maximizing packet delivery ratio (PDR) and throughput (Th) and minimizing delay (D) and number of control packets (C).

This requires integrating between the network simulation and the optimization process to evaluate the network performance response for a specific time interval $(t)$ in respect to each available solution.

A weighted sum is used to aggregate these multiple objectives into a single objective function as

$$
\begin{aligned}
& \text { Maximize } \quad F\left(x_{i}\right)=w_{1} \cdot f_{P D R}\left(x_{i}\right)+w_{2} \cdot f_{T h}\left(x_{i}\right) \\
& +w_{3} \cdot \frac{1}{f_{D}\left(x_{i}\right)}+w_{4} \cdot \frac{1}{f_{C}\left(x_{i}\right)}
\end{aligned}
$$

where each individual objective factor term is qualified as a ratio between theobtained performance metric in response to the applied solution $\left(x_{i}\right)$ with respect to the original achieved result by means of the traditional protocol, in order to quantify the efficiency of change in each evaluation term under using the available solution model in the proposed routing protocol as

$$
\begin{aligned}
& f_{P D R}\left(x_{i}\right)=\frac{\left.P D R_{O F A O D V}\left(x_{i}\right)\right|_{t=0} ^{t=100 s}}{\left.P D R_{A O D V}\right|_{t=0} ^{t=100 s}} \\
& f_{\text {Th }}\left(x_{i}\right)=\frac{\text { THROUGHPUT }\left._{\text {OFAODV }}\left(x_{i}\right)\right|_{t=0} ^{t=100 s}}{\text { THROUGHPUT }} \\
& f_{D}\left(x_{i}\right)=\frac{\text { MEAN DELAY }\left.{ }_{\text {OFAODV }}\left(x_{i}\right)\right|_{t=0} ^{t=100 s}}{\text { MEAN DELAY }} \\
& f_{C}\left(x_{i}\right)
\end{aligned}
$$

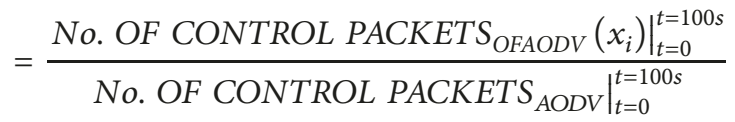

Meanwhile, in light of the facts that the four performance metrics are equally substantial and all the measured factors fall within the same range, 0.25 has been assigned to each weight value $\left(w_{k}\right)$.

\section{Proposed Routing Scheme}

At first assume that each vehicle is equipped with GPS navigation system facility $[27,28]$ to get updated information about vehicle position, road map, and traffic information, besides multiplanned destination directions. Moreover, assume an array antenna $[29,30]$ is utilized in each vehicle to get location and angle of movements of neighbor vehicles.

Apart from assumption issue, the proposed routing scheme Optimized Fuzzy AODV (OFAODV) is looking up 


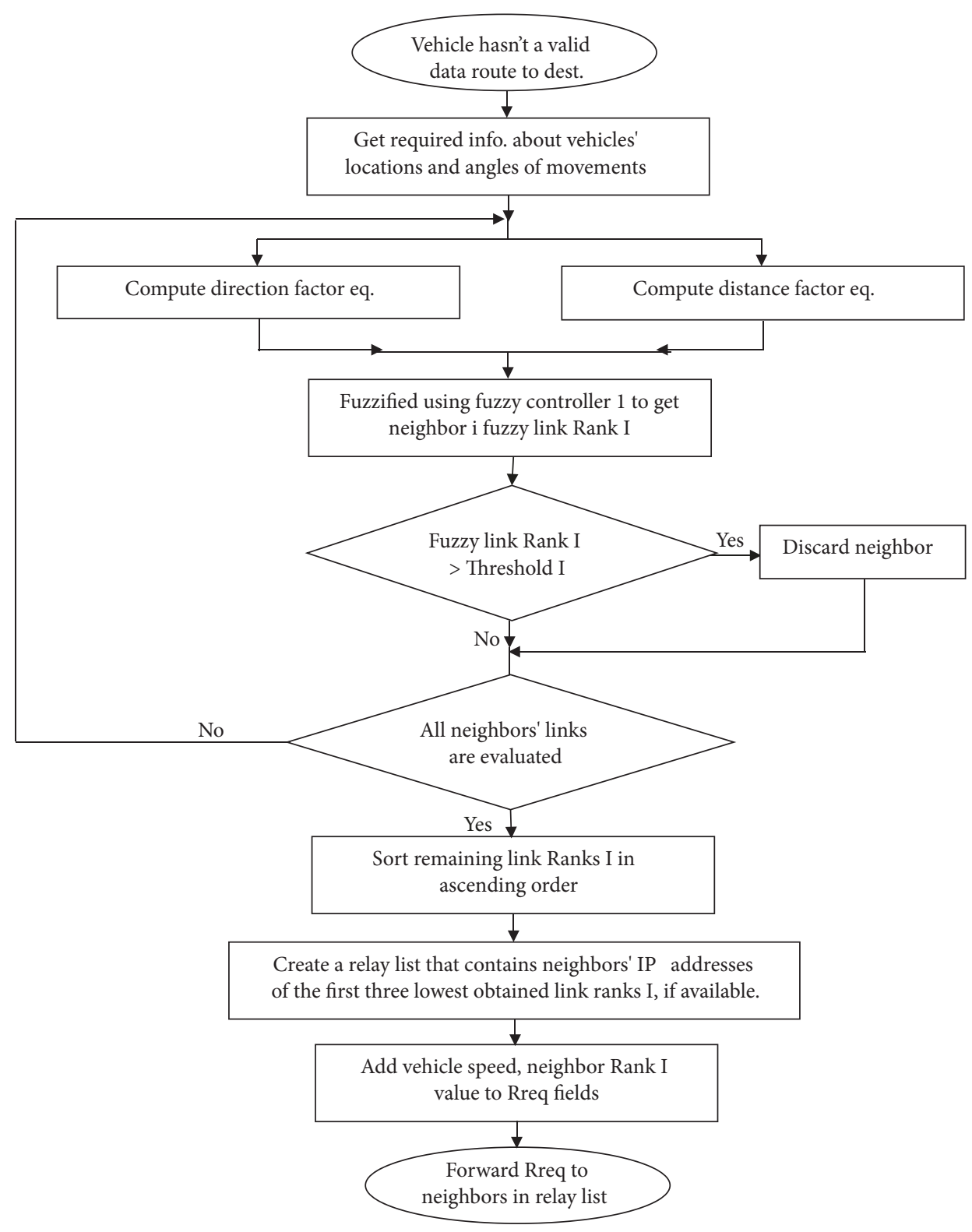

FIgURE 3: Additional steps of OFAODV before RREQ send/forward.

to tune the most used routing protocol in literature: AODV routing protocol $[31,32]$ within three stages during the route discovery phase. The first two stages are carried out before sending the (RREQ) packet, while the last stage is regarding specifying the selected route lifetime in the route reply packet.

In the first modification stage (Figure 3), each vehicle evaluates every one-hop neighbor link rank (Rank I) before attempting to send the RREQ packet using fuzzy logic that combines two factors of direction of neighbor vehicle with respect to destination direction, besides distance factor, which is calculated as a ratio between next node distance to destination as a ratio to current node distance to destination as follows:

\section{Direction Factor}

$$
\begin{aligned}
& =\frac{\mid \text { Neighbor Direction Angle }- \text { Dest.Direction Angle } \mid}{180^{\circ}} \\
& \text { Distance Factor }=\frac{\text { Distance from Neighbor to Dest. }}{\text { Distance from Current node to Dest. }}
\end{aligned}
$$

Consequently, up to only three neighbors with the fewest link rank are specified to be as relay nodes, only if their obtained links' rank is less than a particular value of Rank1_High (Threshold I).

The second tuning stage (Figure 4) starts upon arriving the RREQ packet to the relay node, which assesses Rank 


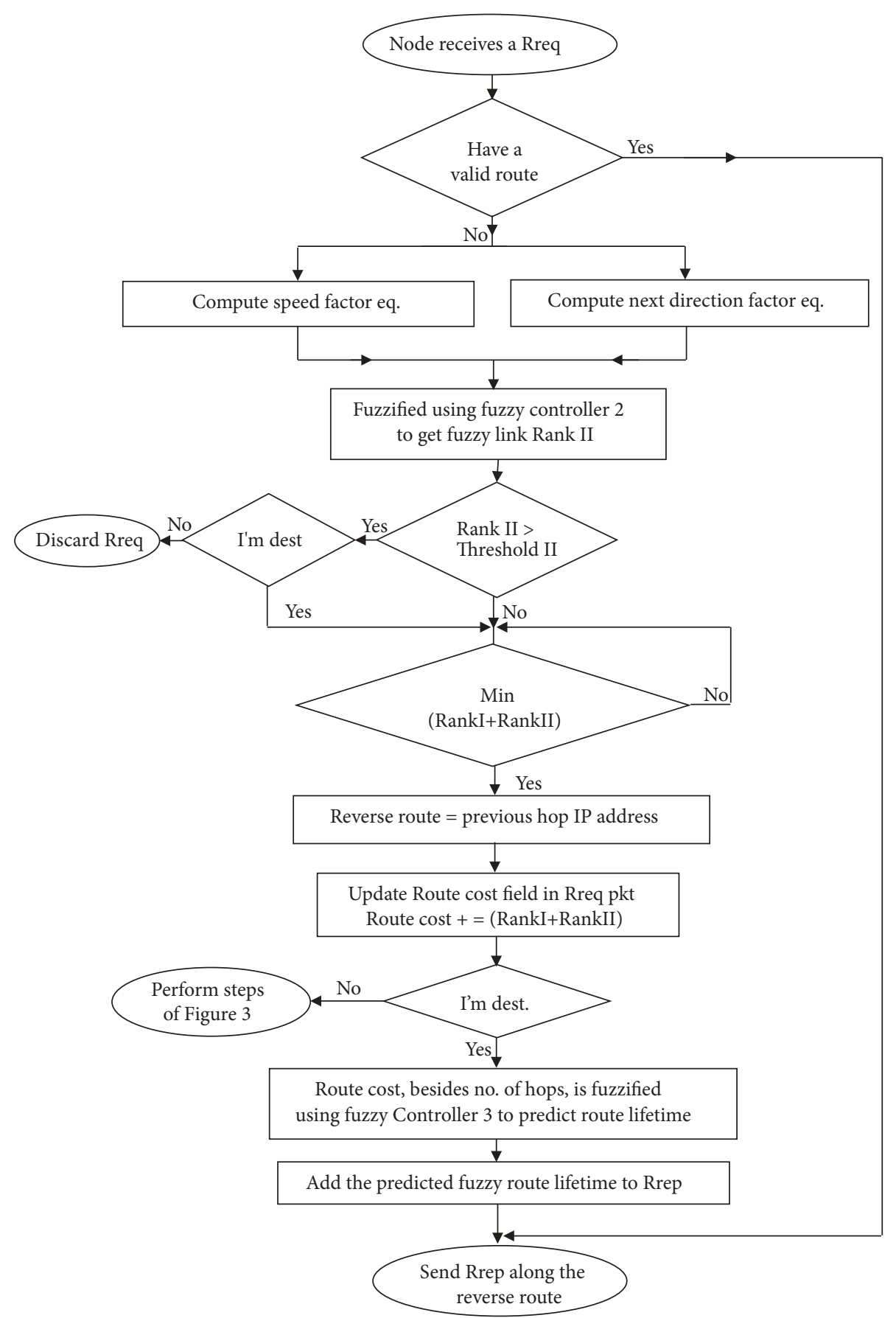

FIGURE 4: Additional steps of OFAODV upon RREQ receiving.

II value of link cost using other fuzzy system joints, other two factors of change in next direction of that vehicle and speed variance between current and previous vehicle as follows:

$$
\begin{aligned}
\text { Next Direction Factor } & =\frac{\mid \text { Node Next Direction Angle }- \text { Node Current Direction Angle } \mid}{180^{0}} \\
\text { Speed Factor } & =\frac{\mid \text { Node Speed }- \text { Previous Node Speed } \mid}{\text { Max Allowable Speed }}
\end{aligned}
$$




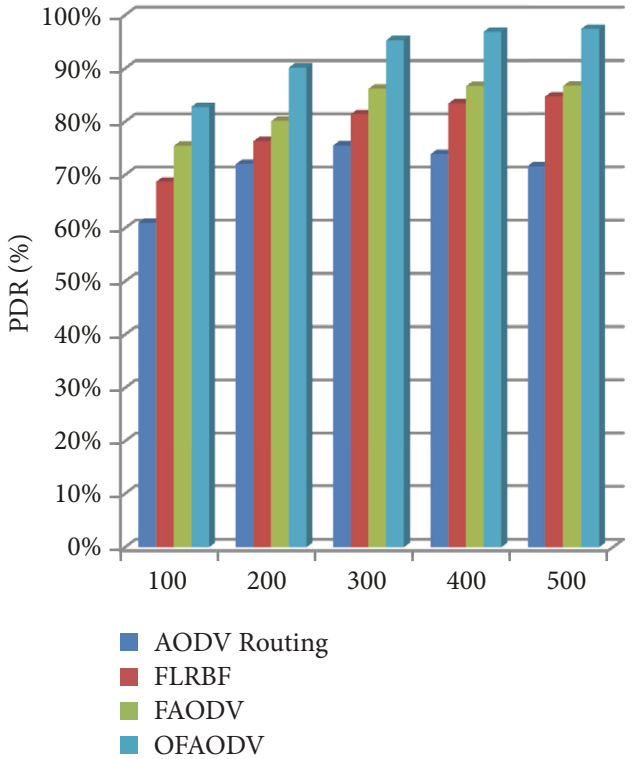

(a) City scenario

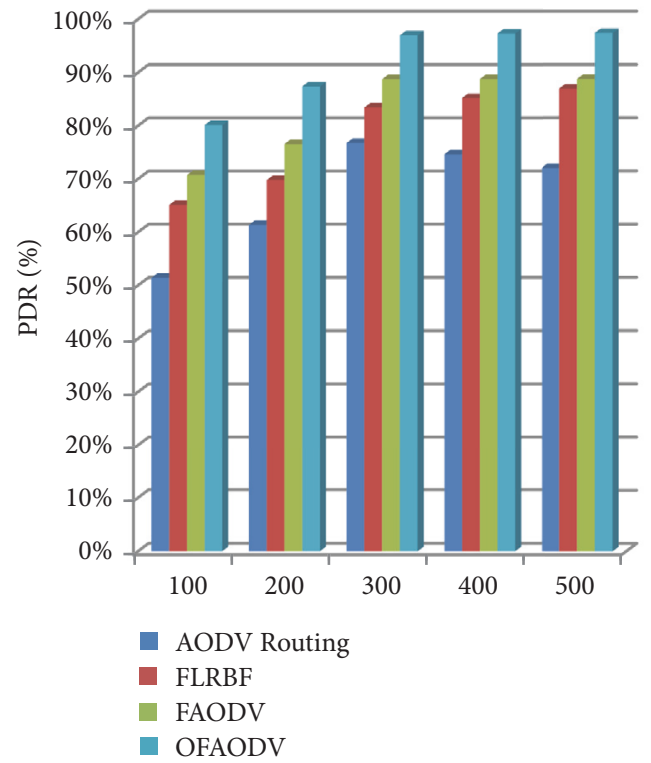

(b) Highway scenario

Figure 5: Packet delivery ratio (\%) vs. no. of vehicles.

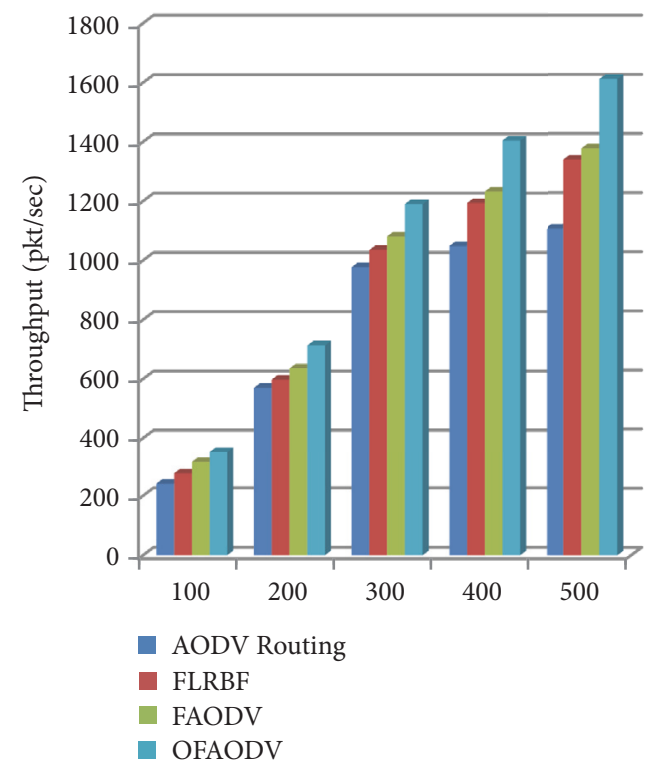

(a) City scenario

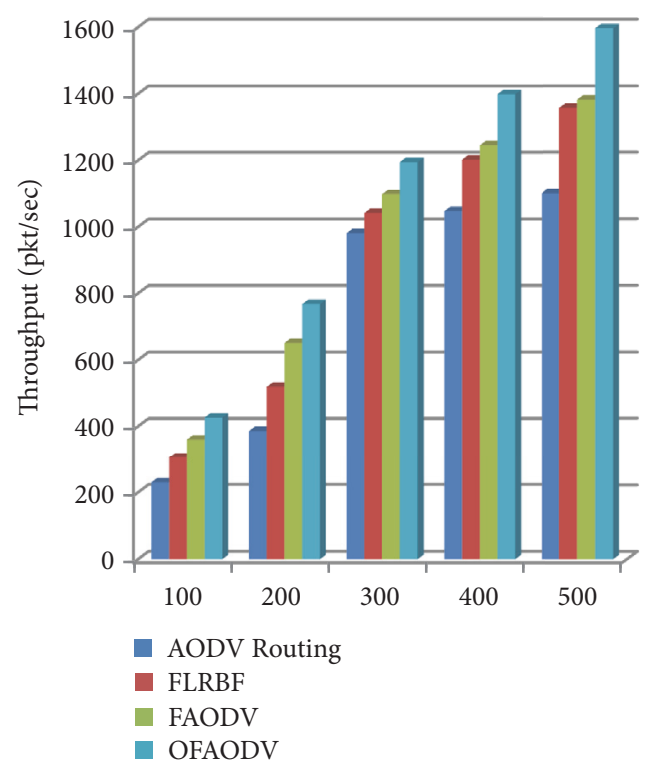

(b) Highway scenario

FIGURE 6: Throughput (pkt/sec) vs. no. of vehicles.

The RREQ packet will be forwarded only if the obtained Rank II value of the link cost is less than a specific value of Rank2_High (Threshold II).

Moreover, the link with the fewest integrated Rank I plus Rank II cost will be selected to construct the optimal route to the destination.

Last modification stage executed before the destination sends the route reply packet, and the information of route cost and number of hops in the received RREQ packet are incorporated using fuzzy logic to predict the selected route lifetime.
Furthermore, the values of Rank1_High, Rank2_High, all fuzzy membership functions' vertices, and fuzzy rules are optimized using $A B C$ algorithm (as described in previous section) aiming to improve the most important network performance outcomes.

\section{Simulation Model and Results}

A simulated area of $3 \mathrm{~km}$ by $3 \mathrm{~km}$ of real maps of Basra city and 195 highway is generated using bidirectional coupling of 


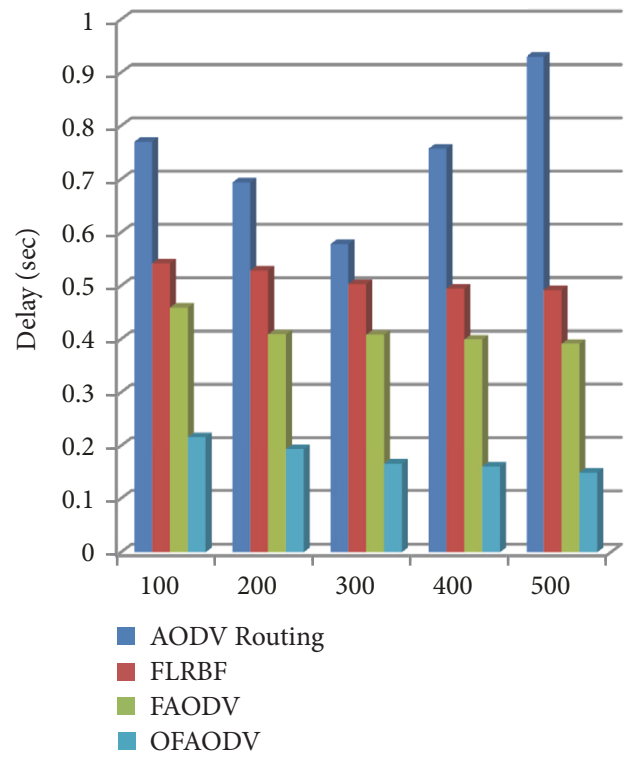

(a) City scenario

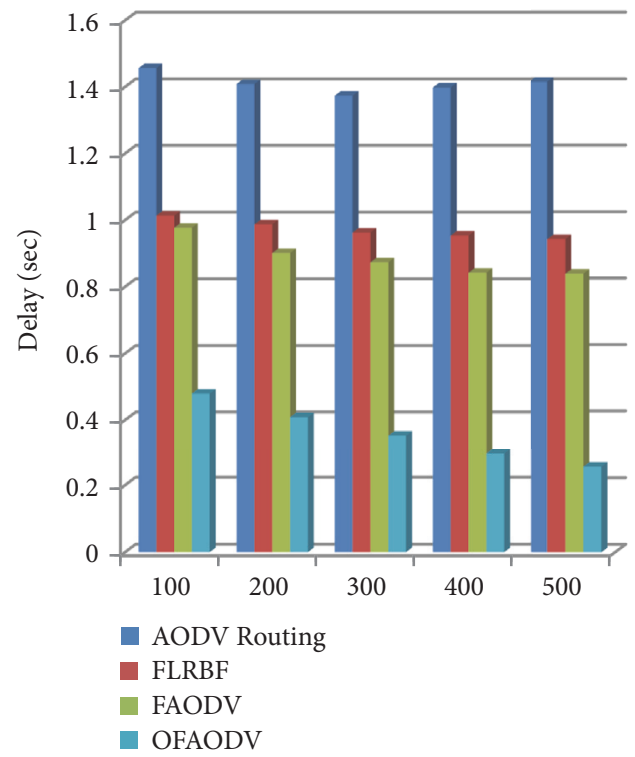

(b) Highway scenario

Figure 7: Mean delay (Sec) vs. no. of vehicles.

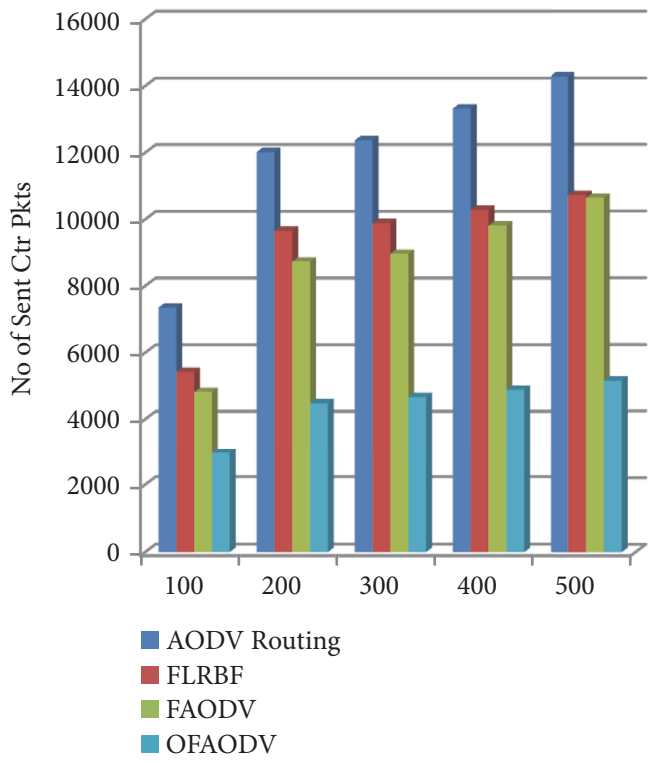

(a) City scenario

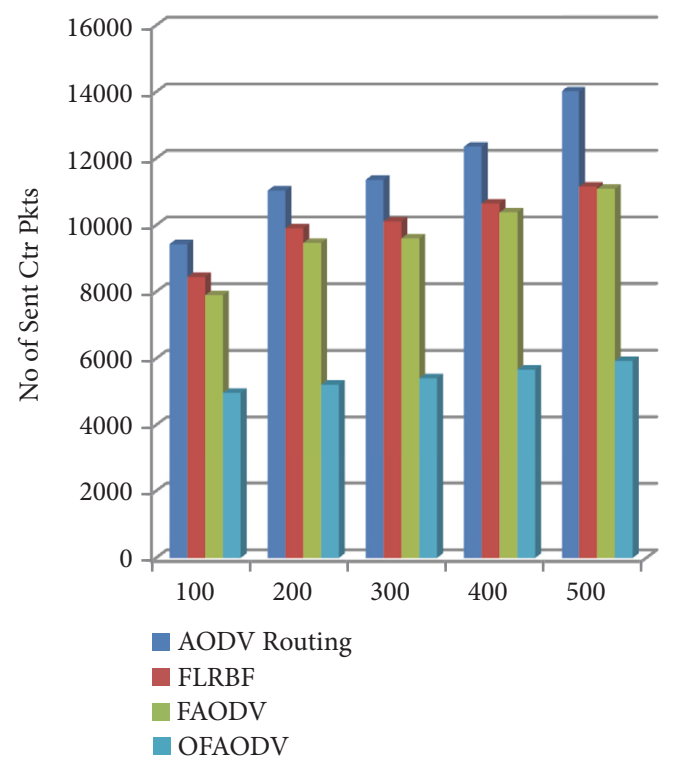

(b) Highway scenario

Figure 8: No. of sent control packets vs. no. of vehicles.

SUMO and OMNeT++ simulators to allow direct interaction between vehicular traffic dynamics and network communication system. Along 1000 simulated seconds, a number of 100 , $200,300,400$, and 500 vehicles are simulated to move within each simulated map with TraCI mobility model, each vehicle has a transmission range of $250 \mathrm{~m}$, IEEE 802.11p was used as a medium access control protocol, and UDP data traffic was generated with 512B in size for each sent packets.

On the other hand, the present proposed routing protocol (OFAODV) has been simulated and compared with other three protocols: AODV routing protocol, Fuzzy Logic
Routing Based on forwarding optimization (FLRBF) [9], and Fuzzy based AODV routing (FAODV), whereby three criteria regarding number of relay vehicles, distance, and speed of movement of vehicles are considered in route selection decision using fuzzy logic.

The performance evaluation of all the simulated protocols is carried out in terms of packet delivery ratio (Figure 5), throughput (Figure 6), mean delay (Figure 7), and number of sent control packets (Figure 8).

The obtained results have clearly demonstrated the significant improvement of network performance when using 
the proposed method (OFAODV routing) by an average of $23.15 \%, 45.77 \%, 75.15 \%$, and $57.65 \%$ over the traditional AODV protocol in PDR, throughput, delay, and number of sent ctrl packets, respectively as compared with $13.84 \%$, $27.56 \%, 40.22 \%$, and $22.43 \%$, percentage of achieved improvement by means of FAODV protocol, and an upgrading average of $9.47 \%, 17.14 \%, 30.38 \%$, and $18 \%$ under FLRBF protocol.

\section{Conclusion}

The present work introduces an optimal routing method that eliminates the dissemination of RREQ packets; also it could reduce routes error and contention overhead by augmenting the efficiency of the selected routes, with the ability to predict their optimal lifetime.

In order to accommodate that, three two-inputs fuzzy systems are employed based on multiple criteria of distance, direction, speed, future direction, and number of hops information.

Furthermore, artificial bee colony optimization algorithm is used to automatically extract these fuzzy systems' memberships and rules, as well as obtaining link cost limitations within which relay nodes are selected.

Simulation results verified that the proposed routing protocol significantly improves the network performance in terms of packet delivery ratio, throughput, end to end delay, and number of control packets in both urban and freeway environments under different conditions of vehicle density.

\section{Data Availability}

The data used to support the findings of this study are available from the corresponding author upon request.

\section{Conflicts of Interest}

The authors declare that they have no conflicts of interest.

\section{References}

[1] A. Laouiti, A. Qayyum, and M Saad, Vehicular Ad-Hoc Networks for Smart Cities, Springer, 2017.

[2] I. A. Abbasi and A. S. Khan, "A review of vehicle to vehicle communication protocols for VANETs in the urban environment," Future Internet, vol. 10, no. 2, 2018.

[3] M. Dixit, R. Kumar, and A. K. Sagar, "VANET: Architectures, research issues, routing protocols, and its applications," in Proceedings of the 2016 IEEE International Conference on Computing, Communication and Automation (ICCCA '16), pp. 555-561, April 2016.

[4] M. Jain and R. Saxena, "Overview of VANET: Requirements and its routing protocols," in Proceedings of the 2017 International Conference on Communication and Signal Processing (ICCSP), pp. 1957-1961, Chennai, April 2017.

[5] G. Li, L. Boukhatem, and J. Wu, "Adaptive Quality-of-ServiceBased Routing for Vehicular Ad Hoc Networks with Ant Colony Optimization," IEEE Transactions on Vehicular Technology, vol. 66, no. 4, pp. 3249-3264, 2017.
[6] N. M. Al-Kharasani, Z. A. Zulkarnain, S. Subramaniam, and Z. M. Hanapi, "An efficient framework model for optimizing routing performance in vanets," Sensors, vol. 18, no. 2, 2018.

[7] S. Latif, S. Mahfooz, B. Jan et al., "Multicriteria Based Next Forwarder Selection for Data Dissemination in Vehicular Ad Hoc Networks Using Analytical Network Process," Mathematical Problems in Engineering, vol. 2017, Article ID 4671892, 18 pages, 2017.

[8] X. Zhang, X. Zhang, and C. Gu, "A micro-artificial bee colony based multicast routing in vehicular ad hoc networks," Ad Hoc Networks, vol. 58, pp. 213-221, 2017.

[9] Z. Miao, X. Cai, Q. Luo, and W. Dong, "A FLRBF scheme for optimization of forwarding broadcast packets in vehicular ad hoc networks," in Proceedings of the 27th IEEE Annual International Symposium on Personal, Indoor, and Mobile Radio Communications, PIMRC 2016, Spain, September 2016.

[10] M. Nabil, A. Hajami, and A. Haqiq, "A stable route and the remaining time prediction to send a data packet in highway environment," in Proceedings of the 16th International Conference on Hybrid Intelligent Systems, Advances in Intelligent Systems and Computing, vol. 552, pp. 11-20, Springer, 2017.

[11] C. Wu, S. Ohzahata, Y. Ji, and T. Kato, "Joint fuzzy relays and network-coding-based forwarding for multihop broadcasting in vanets," IEEE Transactions on Intelligent Transportation Systems, vol. 16, no. 3, pp. 1415-1427, 2015.

[12] C. Lin, F. Dong, and K. Hirota, "Fuzzy Road Situation Model Optimization Routing (FRSMOR) in Vehicular Ad-hoc Network (VANET)," in Proceedings of the 2012 Joint 6th International Conference on Soft Computing and Intelligent Systems, SCIS 2012 and 13th International Symposium on Advanced Intelligence Systems, ISIS 2012, pp. 532-537, Japan, November 2012.

[13] L. Zhou, G. Cui, H. Liu, Z. Wu, and D. Luo, "NPPB: A broadcast scheme in dense VANETs," Information Technology Journal, vol. 9, no. 2, pp. 247-256, 2010.

[14] S. Shelly, V. Vijay, and A. V. Babu, "Model for path duration in vehicular ad hoc networks under greedy forwarding strategy," in Proceedings of the International Conference on Computer, Communication and Convergence, ICCC 2015, pp. 394-400, India, December 2015.

[15] K. S. Kaswan, S. Choudhary, and K. Sharma, "Applications of artificial Bee Colony Optimization technique: Survey," in Proceedings of the 2nd International Conference on Computing for Sustainable Global Development, INDIACom 2015, pp. 16601664, India, March 2015.

[16] D. Karaboga, B. Gorkemli, C. Ozturk, and N. Karaboga, "A comprehensive survey: artificial bee colony (ABC) algorithm and applications," Artificial Intelligence Review, vol. 42, pp. 2157, 2014.

[17] A. Kumar, D. Kumar, and S. K. Jarial, "A review on artificial bee colony algorithms and their applications to data clustering," Cybernetics and Information Technologies, vol. 17, no. 3, pp. 3-28, 2017.

[18] D. Karaboga and B. Akay, "A comparative study of artificial Bee colony algorithm," Applied Mathematics and Computation, vol. 214, no. 1, pp. 108-132, 2009.

[19] B. Nozohour-leilabady and B. Fazelabdolabadi, "On the application of artificial bee colony (ABC) algorithm for optimization of well placements in fractured reservoirs; efficiency comparison with the particle swarm optimization (PSO) methodology," Petroleum, vol. 2, no. 1, pp. 79-89, 2016. 
[20] M. Konar and A. Bagis, "Performance comparison of particle swarm optimization, differential evolution and artificial bee colony algorithms for fuzzy modelling of nonlinear systems," Elektronika ir Elektrotechnika, vol. 22, no. 5, pp. 8-13, 2016.

[21] J. Kacprzyk, E. Szmidt, S. Zadrozny, K. Atanassov, M. Krawczyk, and S. Zadrożny, Advances in Fuzzy Logic and Technology, Springer, 2017.

[22] A. Zilouchian and M. Jamshidi, Intelligent Control Systems Using Soft Computing Methodologies, CRC Press LLC, 2001.

[23] A. A. Ali and T. O. Fahad, "Fuzzy Energy Based Routing Protocol for MANET,' International Journal of Computer Applications, vol. 46, no. 1, pp. 38-40, 2012.

[24] T. O. Fahad and A. A. Ali, "Improvement of AODV Routing on MANETs Using Fuzzy Systems," in Proceedings of the 1st IEEE International Conference of Energy, Power, and Control, pp. 297301, Iraq, 2010.

[25] T. O. Fahad and A. A. Ali, "Fuzzy Controller Based Stable Routes with Lifetime Prediction in MANETs," International Journal of Computer Networks (IJCN), vol. 3, no. 1, pp. 37-42, 2011.

[26] T. O. Fahad and A. A. Ali, "Compressed fuzzy logic based multicriteria aodv routing in VANET environment," in Proceedings of the International Journal of Electrical and Computer Engineering, vol. 9, no. 1, 2019.

[27] W. Rahiman and Z. Zainal, "An overview of development GPS navigation for autonomous car," in Proceedings of the 2013 IEEE 8th Conference on Industrial Electronics and Applications, ICIEA 2013, pp. 1112-1118, Australia, June 2013.

[28] P. G. Parakkal and V. V. Sajith Variyar, "GPS based navigation system for autonomous car," in Proceedings of the 2017 International Conference on Advances in Computing, Communications and Informatics, ICACCI 2017, pp. 1888-1893, India, September 2017.

[29] V. Rabinovich and N. Alexandrov, Antenna Arrays and Automotive Applications, Springer, 2013.

[30] M. A. M. Marinho, A. Vinel, F. Antreich, J. P. C. L. Da Costa, and E. P. De Freitas, "Antenna Array Based Localization Scheme for Vehicular Networks," in Proceedings of the 17th IEEE International Conference on Computer and Information Technology, CIT 2017, pp. 142-146, Finland, August 2017.

[31] A. Gupta, R. Singh, D. Ather, and R. S. Shukla, "Comparison of various routing algorithms for VANETS," in Proceedings of the 5th International Conference on System Modeling and Advancement in Research Trends, SMART 2016, pp. 153-157, India, November 2016.

[32] C. Perkins, E. Belding-Royer, and S. Das, "Ad hoc on-demand distance vector (AODV) routing," No. RFC 3561, 2003. 


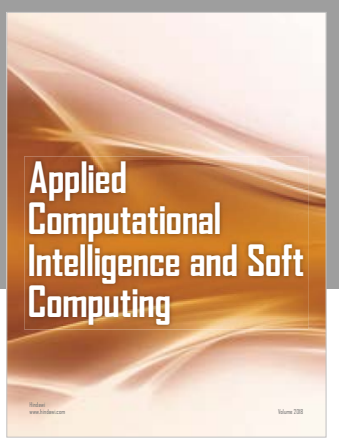

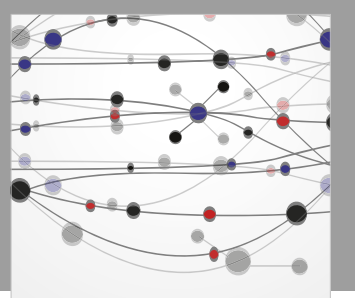

The Scientific World Journal
Submit your manuscripts at

Computing
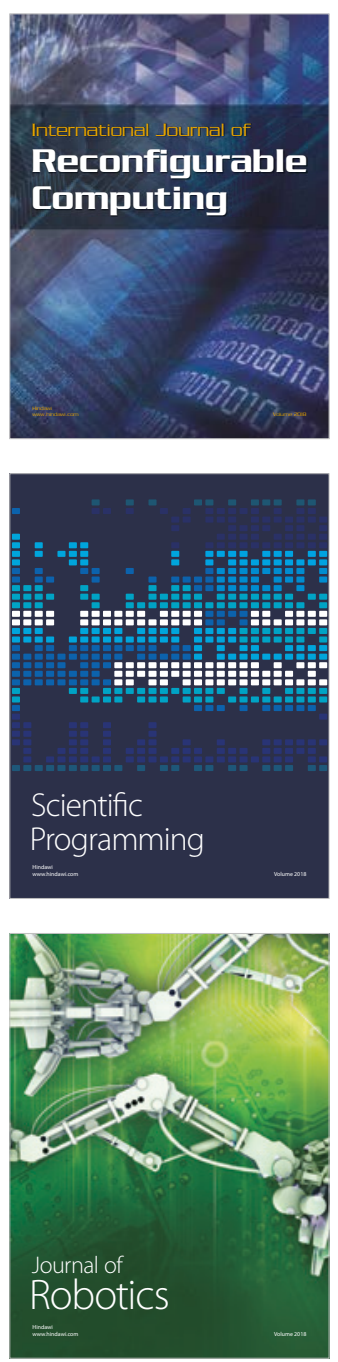

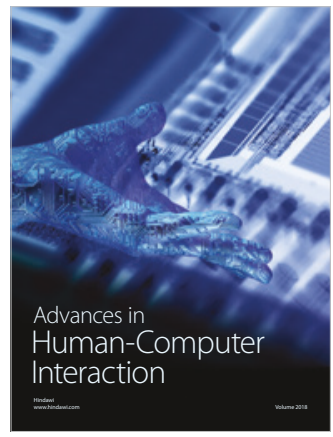

Human-Compute

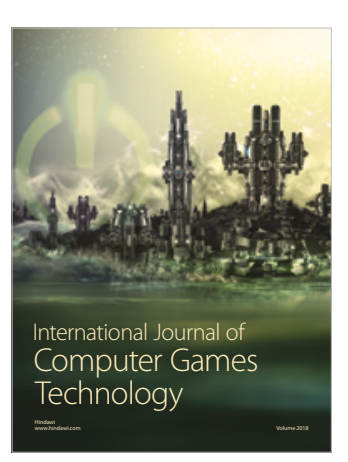

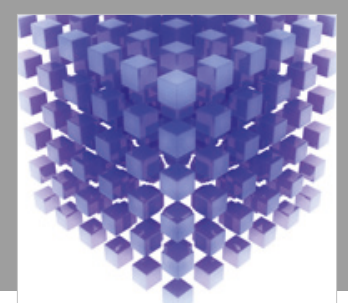

Mathematical Problems in Engineering

\section{Engincering}
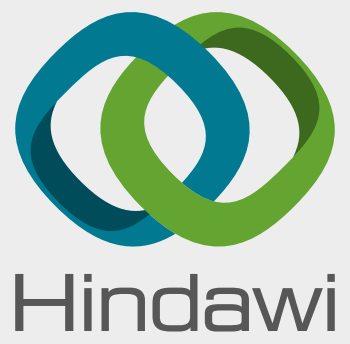

www.hindawi.com
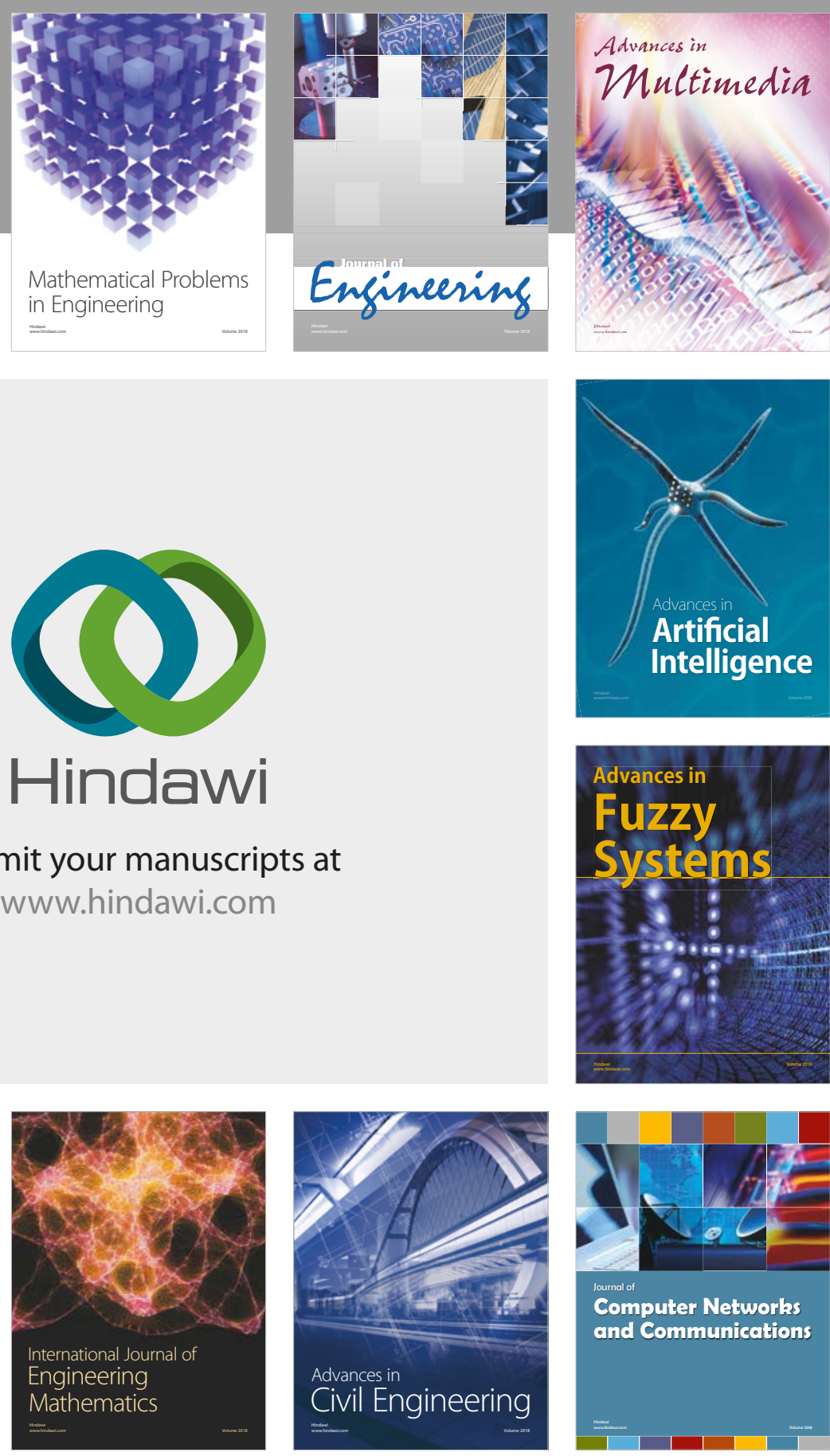

Computer Networks and Communications

Multimedia
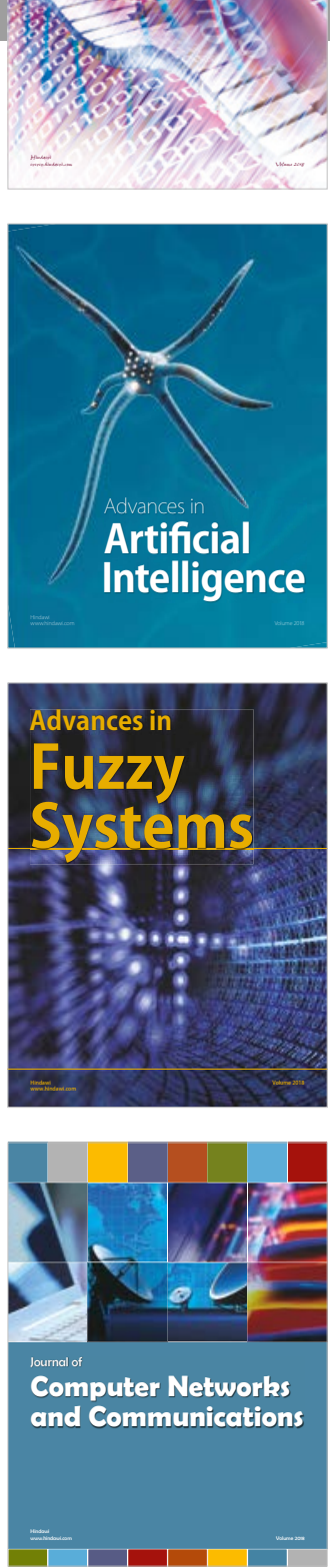

Advances in

Modelling \&

Simulation

in Engineering

interaction

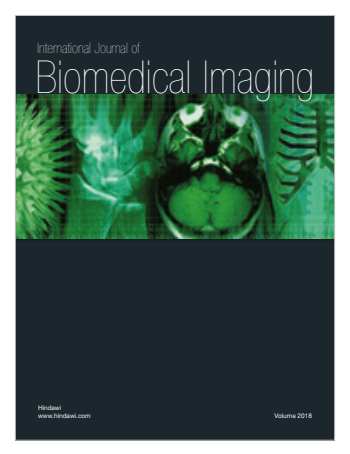

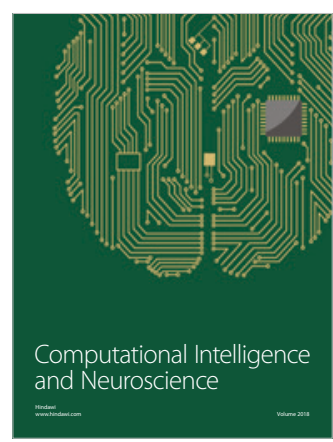

